\title{
Recursive integral method for transmission eigenvalues
}

\author{
Ruihao Huang * $^{*} \quad$ Allan A. Struthers ${ }^{\dagger} \quad$ Jiguang Sun ${ }^{\ddagger} \quad$ Ruming Zhang $\S$
}

\begin{abstract}
Transmission eigenvalue problems arise from inverse scattering theory for inhomogeneous media. These non-selfadjoint problems are numerically challenging because of a complicated spectrum. In this paper, we propose a novel recursive contour integral method for matrix eigenvalue problems from finite element discretizations of transmission eigenvalue problems. The technique tests (using an approximate spectral projection) if a region contains eigenvalues. Regions that contain eigenvalues are subdivided and tested recursively until eigenvalues are isolated with a specified precision. The method is fully parallel and requires no a priori spectral information. Numerical examples show the method is effective and robust.
\end{abstract}

\section{Introduction}

The non-selfadjoint transmission eigenvalue problem $[8,5,25,6]$ has important applications in inverse scattering for inhomogeneous media. Sampling methods for reconstructing the support of an inhomogeneous medium fail if the interrogating frequency corresponds to a transmission eigenvalue [6]. Early studies focused on showing that transmission eigenvalues are at most a discrete point set. Later, it was realized that transmission eigenvalues can be extracted from the scattering data and used to reconstruct physical properties of unknown targets [5].

Recently, significant efforts have been devoted to develop numerical methods for transmission eigenvalues [9, 26, 15, 34, 28, 1, 18, 7, 20, 33, 29]. Colton, Monk, and Sun [9] proposed three finite element methods. Ji et al. [15] developed a mixed method based on a fourth order formulation. An and Shen [1] proposed an efficient spectral-element method for two-dimensional radially-stratified media. Sun [26] introduced a conforming finite element method where real transmission eigenvalues are computed as roots of a nonlinear function generated by a related fourth order problem. Cakoni et al. [7] proposed a new mixed finite element method and proved convergence based on Osborn's theory [22]. Li et al. [20] developed a finite element method for a quadratic eigenvalue problem. Integral equations are also used to compute transmission eigenvalues. Cossonniére and Haddar[10] formulated the transmission eigenvalue problem as a nonlinear integral eigenvalue problem (see also [35]). Kleefeld [18] adapted the spectral projection based technique proposed by Beyn [3] to the

\footnotetext{
*Department of Mathematical Sciences, Michigan Technological University, Houghton, MI 49931 (ruihaoh@mtu.edu).

${ }^{\dagger}$ Department of Mathematical Sciences, Michigan Technological University, Houghton, MI 49931 (struther@mtu.edu).

${ }^{\ddagger}$ Department of Mathematical Sciences, Michigan Technological University, Houghton, MI 49931 (jiguangs@mtu.edu).

${ }_{\S}^{\S}$ Department of Mathematical Sciences, Michigan Technological University, Houghton, MI 49931 (rumingz@mtu.edu).
}

(C) 2016. This manuscript version is made available under the Elsevier user license http://www.elsevier.com/open-access/userlicense/1.0/ 


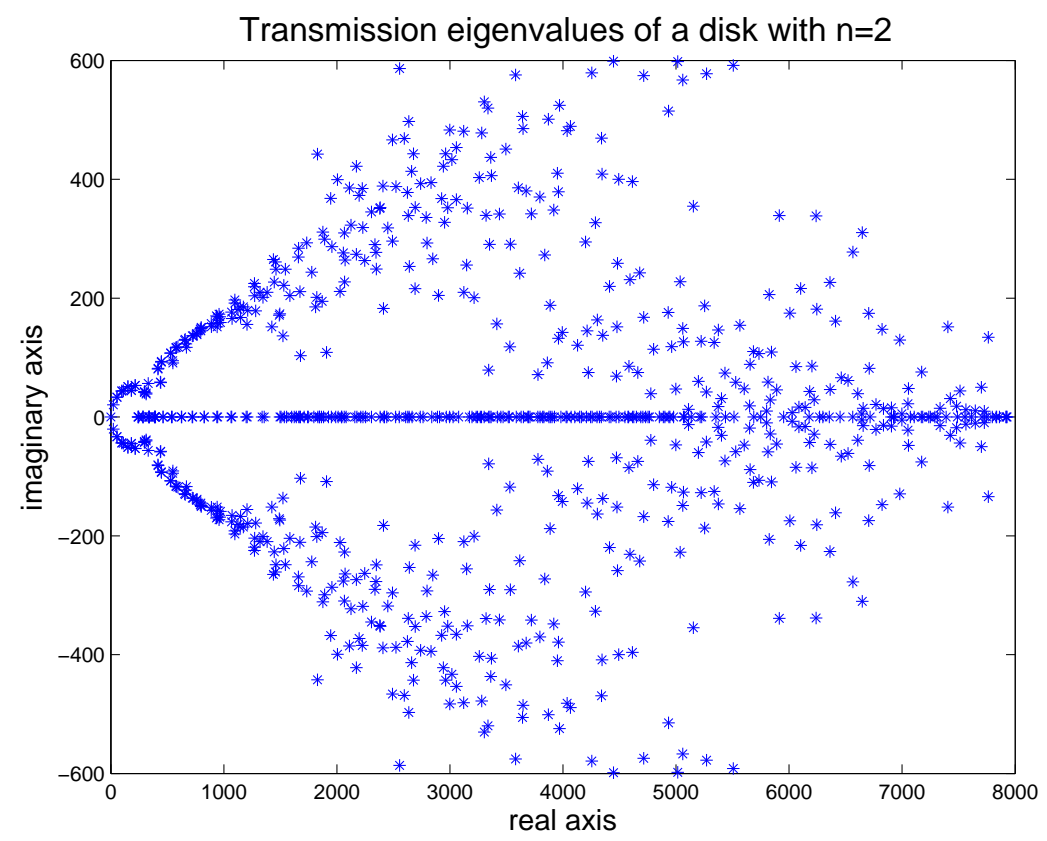

Figure 1: Transmission eigenvalues on the complex plane: a disk with radius $1 / 2$ and index of refraction $n=2$.

this integral formulation. Non-traditional methods, including linear sampling [27] and inside-out duality [19] have also been used to extract eigenvalues from scattering data. The reader is referred to other methods in $[11,14,16,12,34]$ for the transmission eigenvalue and related source problems.

Most existing eigenvalue solvers are not well adapted for finite element discretizations of transmission eigenvalue problems. In general, physical interest is focused on eigenvalues in the interior of a complex spectra (see Fig. 1) of a large (but sparse) non-Hermitian (the underlying continuous problem is non-selfadjoint) generalized matrix eigenvalue problem..

In this paper, we propose a novel recursive integral method (RIM) to approximate the generalized matrix eigenvalues arising from finite element discretizations of transmission eigenvalue problems. The aim is to develop a general eigensolver capable of resolving interior eigenvalues within a specified rectangle in the complex plane for non-selfadjoint problems with complicated spectra for which no a priori spectral information is available.

Contour integral based spectral projection is a classical tool in operator theory [17] which has recently been adapted to approximate invariant subspaces [24, 23, 3, 31, 32, 13, 2] corresponding to the eigenvalues within a given simple closed curve in the complex plane. The original large eigenvalue problem is reduced to a much smaller problem on the computed approximate subspace.

In contrast, our RIM algorithm tests a rectangular region in the complex plane (using an approximate spectral projection) for eigenvalues. If eigenvalue(s) are detected the region is recursively subdivided and tested until their diameter is less than some specified tolerance. Note, the primary distinction between RIM and earlier contour integral based algorithms is that RIM isolates eigenvalues in small sub-domains without solving smaller projected eigenproblems. 
The paper is arranged as follows. Section 2 introduces the transmission eigenvalue problem and a mixed finite element approximation (using linear Lagrange elements) which generates our generalized non-Hermitian matrix eigenvalue test problems. Section 3 introduces RIM. Section 4 discusses some implementation details. Section 5 contains numerical results on some test problems. Section 6 concludes with some discussion and proposed future work.

\section{Transmission eigenvalue problem}

Let $D \subset \mathbb{R}^{d}, d=2,3$, be an open bounded domain with Lipschitz boundary $\partial D$ and outward unit normal $\nu$. The direct scattering problem for the incident plane wave in direction $p\left(p \in \mathbb{R}^{d}\right.$ with $|p|=1$ ) and wave number $k$ by an inhomogeneous medium occupying $D$ (with index of refraction $n(x)$ satisfy $n(x) \geq n_{0}>1$ for $\left.x \in D\right)$ is to determine the total field $u(x)$ satisfying

$$
\begin{array}{lr}
\Delta u+k^{2} n(x) u=0, & \text { in } D, \\
\Delta u+k^{2} u=0, & \text { in } \mathbb{R}^{d} \backslash D, \\
u(x)=e^{i k x \cdot p}+u^{s}(x), & \text { in } \mathbb{R}^{d}, \\
\lim _{r \rightarrow \infty} \sqrt{r}\left(\frac{\partial u^{s}}{\partial r}-i k u^{s}\right)=0 . &
\end{array}
$$

The incident plane wave is $u^{i}=e^{i k x \cdot p}$ and the scattered field $u^{s}$ satisfies the Sommerfeld radiation condition (1d) uniformly with respect to $\hat{x}=x / r, r=|x|$.

The transmission eigenvalue problem is to find $\lambda:=k^{2} \in \mathbb{C}$ and non-trivial $w$ and $v$ satisfying

$$
\begin{array}{lr}
\Delta w+\lambda n(x) w=0, & \text { in } D, \\
\Delta v+\lambda v=0, & \text { in } D, \\
w-v=0, & \text { on } \partial D, \\
\frac{\partial w}{\partial \nu}-\frac{\partial v}{\partial \nu}=0, & \text { on } \partial D .
\end{array}
$$

The problem is readily transformed into a fourth order problem in $z=v-w \in H_{0}^{2}(D)$ by subtracting $(2 \mathrm{a})$ from $(2 \mathrm{~b})$ to get

$$
(\Delta+\lambda n(x)) z=-\lambda(n(x)-1) v .
$$

Dividing by $n(x)-1$ and applying $(\Delta+\lambda)$ gives the fourth order equation

$$
(\Delta+\lambda)\left(\frac{1}{n(x)-1}(\triangle+\lambda n(x))\right) z=0 .
$$

Defining $y=\frac{1}{n(x)-1}(\Delta+\lambda n(x)) z$ gives the mixed formulation

$$
\begin{aligned}
(\Delta+\lambda) y & =0 \\
\frac{1}{n(x)-1}(\Delta+\lambda n(x)) z & =y
\end{aligned}
$$


The associated weak mixed problem is to find $(\lambda, z, y) \in \mathbb{C} \times H_{0}^{1}(D) \times H^{1}(D)$ satisfying

$$
\begin{aligned}
(\nabla y, \nabla \phi) & =\lambda(y, \phi) \quad \text { for all } \phi \in H_{0}^{1}(D), \\
(\nabla z, \nabla \varphi)+((n(x)-1) y, \varphi) & =\lambda(n(x) z, \varphi) \text { for all } \varphi \in H^{1}(D) .
\end{aligned}
$$

To describe the implementation proposed in [15] of this mixed method for linear Lagrange elements on a triangular mesh for $D \subset \mathbb{R}^{2}$ or a tetrahedral mesh for $D \subset \mathbb{R}^{3}$ we simply define the linear Lagrange finite element spaces

$$
\begin{aligned}
V_{h} & =\text { linear Lagrange elements on } D \\
V_{h}^{0} & =V_{h} \cap H_{0}^{1}(D) \\
& =\text { functions in } V_{h} \text { which are zero on } \partial D, \\
V_{h}^{\mathcal{B}} & =V_{h} \backslash V_{h}^{0} \text { functions in } V_{h} \text { which are zero within } D .
\end{aligned}
$$

The discrete weak formulation is to find $\left(\lambda_{h}, z_{h}, y_{h}\right) \in \mathbb{C} \times V_{h}^{0} \times V_{h}$ such that

$$
\begin{aligned}
\left(\nabla y_{h}, \nabla \phi_{h}\right) & =\lambda_{h}\left(y_{h}, \phi_{h}\right) \quad \text { for all } \phi_{h} \in V_{h}^{0}, \\
\left(\nabla z_{h}, \nabla \varphi_{h}\right)+\left((n(x)-1) y_{h}, \varphi_{h}\right) & =\lambda_{h}\left(n(x) z_{h}, \varphi_{h}\right) \quad \text { for all } \varphi_{h} \in V_{h} .
\end{aligned}
$$

Let $\psi_{1}, \ldots, \psi_{K}$ be a basis for $V_{h}^{0}$ and $\psi_{1}, \ldots, \psi_{K}, \psi_{K+1}, \ldots, \psi_{T}$ be a basis for $V_{h}$ such that $z_{h}=\sum_{i=1}^{K} z_{i} \psi_{i}$ and $y_{h}=\sum_{i=1}^{T} y_{i} \psi_{i}$. Define the column (' denotes the transpose) vectors $\boldsymbol{z}=$ $\left(z_{1}, \ldots, z_{K}\right)^{\prime}$ and $\boldsymbol{y}=\left(y_{1}, \ldots, y_{T}\right)^{\prime}$ to write the matrix problem as

$$
\begin{aligned}
S_{K \times T} \boldsymbol{y} & =\lambda_{h} M_{K \times T} \boldsymbol{y}, \\
S_{T \times K} \boldsymbol{z}+M_{T \times T}^{n(x)-1} \boldsymbol{y} & =\lambda_{h} M_{T \times K}^{n(x)} \boldsymbol{z},
\end{aligned}
$$

where

$$
\begin{aligned}
\left(S_{K \times T}\right)_{i, j} & =\left(\nabla \psi_{i}, \nabla \psi_{j}\right), \quad 1 \leq i \leq K, 1 \leq j \leq T \\
\left(S_{T \times K}\right)_{i, j} & =\left(\nabla \psi_{i}, \nabla \psi_{j}\right), \quad 1 \leq i \leq T, 1 \leq j \leq K \\
\left(M_{K \times T}\right)_{i, j} & =\left(\psi_{i}, \psi_{j}\right), \quad 1 \leq i \leq K, 1 \leq j \leq T \\
\left(M_{T \times K}^{n(x)}\right)_{i, j} & =\left(n(x) \psi_{i}, \psi_{j}\right), \quad 1 \leq i \leq T, 1 \leq j \leq K, \\
\left(M_{T \times T}^{n(x)-1}\right)_{i, j} & =\left((n(x)-1) \psi_{i}, \psi_{j}\right), \quad 1 \leq i \leq T, 1 \leq j \leq T .
\end{aligned}
$$

The generalized matrix eigenvalue problem that results is

$$
A \boldsymbol{x}=\lambda B \boldsymbol{x}
$$

where non-symmetric $A$ and symmetric $B$ are defined by

$$
A=\left(\begin{array}{cc}
S_{K \times T} & 0_{K \times K} \\
M_{T \times T}^{n(x)-1} & S_{T \times K}
\end{array}\right), \quad B=\left(\begin{array}{cc}
M_{K \times T} & 0_{K \times K} \\
0_{T \times T} & M_{T \times K}^{n(x)}
\end{array}\right)\left(\begin{array}{l}
\boldsymbol{y} \\
\boldsymbol{z}
\end{array}\right), \quad \boldsymbol{x}=\left(\begin{array}{l}
\boldsymbol{y} \\
\boldsymbol{z}
\end{array}\right) .
$$

For simplicity we have dropped the subscript $h$ on the eigenvalue $\lambda$. 


\section{A recursive contour integral method}

This section describes a novel eigensolver for (4) based on approximate spectral projections. We start by recalling some classical spectral theory results from the classic text [17]. For a bounded linear operator $T: \mathcal{X} \rightarrow \mathcal{X}$ on a complex Hilbert space $\mathcal{X}$ the resolvent set of $T$ is

$$
\rho(T)=\left\{z \in \mathbb{C}:(z-T)^{-1} \text { exists as a bounded operator on } \mathcal{X}\right\},
$$

for any $z \in \rho(T)$, the resolvent operator is

$$
R_{z}(T)=(z-T)^{-1}
$$

and the spectrum of $T$ is $\sigma(T)=\mathbb{C} \backslash \rho(T)$. We assume that $T$ has only point spectrum and that the eigenspace associated with each eigenvalue $\lambda \in \sigma(T)$ is finite dimensional. In other words we assume that each $\lambda \in \sigma(T)$ is isolated and that there is a minimal positive integer $\alpha$ satisfying

$$
N\left((\lambda-T)^{\alpha}\right)=N\left((\lambda-T)^{\alpha+1}\right)
$$

where $N$ denotes the null space: the dimension of $N\left((\lambda-T)^{\alpha}\right)$ is the algebraic multiplicity of $\lambda$; $N\left((\lambda-T)^{\alpha}\right)$ is the generalized eigenspace corresponding to $\lambda$; and the geometric multiplicity of $\lambda$ is the dimension of $N(\lambda-T)$.

Let $\Gamma$ be a simple closed curve on the complex plane $\mathbb{C}$ lying in $\rho(T)$ which encloses $m$ eigenvalues $\lambda_{j}, j=1, \ldots, m$. Then the spectral projection $[17]$

$$
P=\frac{1}{2 \pi i} \int_{\Gamma} R_{z}(T) d z
$$

is a projection onto the span of the generalized eigenvectors associated with the enclosed eigenvalues $\lambda_{j}, j=1, \ldots, m$. The projection is determined entirely by the eigenvalues within $\Gamma$ and the associated generalized eigenspace.

The contour integral defining $P \boldsymbol{f}$ is approximated using a simple quadrature rule described by a set of $W$ points $z$ and weights $\omega\left\{z_{j}, \omega_{j}, j=1, \ldots, W\right\}$. For a given $\boldsymbol{f}$

$$
P \boldsymbol{f} \approx \frac{1}{2 \pi i} \sum_{j=1}^{W} \omega_{j} \boldsymbol{x}_{j},
$$

where each $\boldsymbol{x}_{j}$ solves

$$
\left(z_{j}-T\right) \boldsymbol{x}_{j}=\boldsymbol{f}, \quad j=1, \ldots, W .
$$

We write $S$ for the region enclosed by the contour $\Gamma$ and describe how we use our approximation for $P \boldsymbol{f}$ to determine if $S$ contains eigenvalues. Note that with randomly selected $\boldsymbol{f} \in \mathcal{X}$ and probability one $\|P \boldsymbol{f}\|_{\mathcal{X}} \neq 0$ implies $S$ contains at least one eigenvalue while $\|P \boldsymbol{f}\|_{\mathcal{X}}=0$ implies $S$ contains no eigenvalue. We use the quadrature based approximation for $P \boldsymbol{f}$ to select regions which contain eigenvalues. These regions are then recursively split and tested in the same way until the diameter of a region is less than a specified small value. The end result is a collection of small non-overlapping regions which contain all the eigenvalues of $T$ within the contour $\Gamma$. We write $\epsilon$ for the specified diameter of the largest possible remaining region since it serves as a precision 
localizing the eigenvalue(s) within $\epsilon$ of any point in the region. Our computational examples use similar rectangles as regions and we identify all the eigenvalues within a rectangle with the center of the rectangle.

The RIM algorithm is as follows.

$$
\operatorname{RIM}(S, \epsilon, \boldsymbol{f})
$$

Input: region $S$, precision $\epsilon$, randomly chosen $\boldsymbol{f}$

Output: $\lambda$, eigenvalue(s) of $T$ in $S$

1. Approximate $P \boldsymbol{f}$ by (5);

2. Decide if $S$ contains eigenvalue(s) using this approximation for $\|P \boldsymbol{f}\|_{\mathcal{X}}$ :

- If no exit.

- If yes compute the radius of $S$.

- If the radius $>\epsilon$, partition $S$ into subregions $S_{j}, j=1, \ldots N$.

for $j=1$ to $N$

$$
\operatorname{RIM}\left(S_{j}, \epsilon, \boldsymbol{f}\right)
$$

end

- If the radius $\leq \epsilon$, output the eigenvalues and exit.

The next section discusses practical implementation (region shapes, subregion partitioning strategies, quadrature selection, random sample selection, and decision strategies for subdividing regions).

Minor modifications are required to fit the generalized matrix eigenvalue problem (4) (arising from the finite element discretization in Section 2) into this structure. For $z$ in the resolvent set of the matrix pencil $(A, B)$ the resolvent is

$$
R_{z}(A, B)=(z B-A)^{-1}
$$

and the projection onto the generalized eigenspace corresponding to the eigenvalues enclosed by $\Gamma$ is

$$
P(A, B)=\frac{1}{2 \pi i} \int_{\Gamma}(z B-A)^{-1} d z .
$$

Approximating $P(A, B) \boldsymbol{f}$ is similar to before

$$
P \boldsymbol{f} \approx \frac{1}{2 \pi i} \sum_{j=1}^{W} \omega_{j} \boldsymbol{x}_{j},
$$

where $\boldsymbol{x}_{j}$ 's are the solutions of the following linear systems

$$
\left(z_{j} B-A\right) \boldsymbol{x}_{j}=\boldsymbol{f}, \quad j=1, \ldots, W .
$$

Recall that if there are no eigenvalues inside $\Gamma$ then $P \boldsymbol{f}=\mathbf{0}$ for all $\boldsymbol{f} \in \mathbb{C}^{n}$.

In practice, we do need a threshold $\delta_{0}$ to distinguish between $\|P \boldsymbol{f}\|_{\mathcal{X}} \neq 0$ and $\|P \boldsymbol{f}\|_{\mathcal{X}}=0$. We postpone the discussion to the next section. 


\section{Implementation}

This section discusses our RIM implementation for the transmission eigenvalue test problem. A moderate aspect ratio rectangle aligned with the real and imaginary axes is used for the search region. Higher aspect ratio rectangles could be pre-divided into suitable rectangles. We set a simple numerical threshhold $\delta_{0}$ for our approximation to $\left|P_{S} \boldsymbol{f}\right|$ and declare a rectangle $S$ to be admissible if our approximate value exceeds this threshhold. Admissible rectangles are subdivided into 4 subrectangles (with the same aspect ratio as the original rectangle) by simply splitting each edge at the midpoint.

There are several key points in the implementation:

(1) A suitable quadrature rule for (8).

(2) A suitable solver for (9).

(3) A suitable threshold $\delta_{0}$.

We use an extremely coarse quadrature with the midpoints of the four edges of $S$ as quadrature points. The numerical examples show that this is an adequate approximation to the indicator. Note that other contour integral methods use more expensive quadrature e.g. [3] uses twenty-five quadrature points.

For simplicity, the linear systems (9) are solved with the matlab " " command. Other solvers e.g. Isqr are readily substituted and with many opportunities to exploit parallelism in this step.

The threshold $\delta_{0}$ is chosen to balance two effects: The randomly selected $\boldsymbol{f}$ may have only a small component in the range of $P$ (which we write as $\mathcal{R}(P)$ ) in which case $|P \boldsymbol{f}|$ may be small even when there are eigenvalues in $S$; The coarse quadrature used to approximate $|P \boldsymbol{f}|$ means that our indicator will not be zero (and may not even be that small) when there are no eigenvalues in $S$. Our strategy selects the threshold as $\delta_{0}=0.1$ based on substantial experimentation. This high threshold for discarding a region systematically leans towards further investigation of regions that may potentially contain eigenvalues.

To understand the first issue consider an orthonormal basis let $\phi_{j}, j=1, \ldots, M$, for $\mathcal{R}(P)$ which coincides with the eigenspace associated with all the eigenvalues within $S$. For a random $\boldsymbol{f}$

$$
|P \boldsymbol{f}|=|\boldsymbol{f}|_{\mathcal{R}(P)}|=| \sum_{j=1}^{M} a_{j} \phi_{j} \mid=\sum_{j=1}^{M} a_{i}^{2}
$$

where $a_{j}=\left(\boldsymbol{f}, \phi_{j}\right)$. Our solution is to normalize our approximation to $P \boldsymbol{f}$ and approximately project again. The indicator $\delta_{S}$ is set to the approximation to

$$
\left|P\left(\frac{P \boldsymbol{f}}{|P \boldsymbol{f}|}\right)\right| \text {. }
$$

Remark 4.1. Analytically, $P^{2} \boldsymbol{f}=P \boldsymbol{f}$ but the approximations to $P^{2} \boldsymbol{f}$ and $P \boldsymbol{f}$ differ significantly.

The second issue exists if eigenvalue(s) lies just outside $S$ which is inevitable as RIM zooms in on an eigenvalue. Fortunately, RIM has an interesting self-correction property which we will illustrate in the next section.

Here are some details of the implementation: 
1. A rectangular search region $S$.

2. Matlab "\" as the linear solves.

3. One quadrature point for each edge of $S$.

4. One normalized random vector $\boldsymbol{f}$.

5. Projections are computed twice using (11).

6. $\delta_{0}=0.1$, i.e., if $\delta_{S}>0.1, S$ is admissible.

The matrix version of RIM is as follows.

$\operatorname{M-RIM}\left(A, B, S, \epsilon, \delta_{0}, \boldsymbol{f}\right)$

Input: matrices $A, B$, region $S$, precision $\epsilon$, thresh hold $\delta_{0}$, random vector $\boldsymbol{f}$.

Output: generalized eigenvalue(s) $\lambda$ inside $S$

1. Compute $\delta_{S}$ using (11).

2. Decide if $S$ contains eigenvalue(s).

- If $\delta_{S}<\delta_{0}$ then exit.

- Otherwise, compute the diameter of $S$.

- If diameter $>\epsilon$, partition $S$ into subregions $S_{j}, j=1, \ldots N$.

for $j=1$ to $N$

$\operatorname{M-RIM}\left(A, B, S_{j}, \epsilon, \delta_{0}, \boldsymbol{f}\right)$.

end

- if diameter $\leq \epsilon$,

set $\lambda$ to be the center of $S$.

output $\lambda$ and exit.

\section{$5 \quad$ Numerical Examples}

We demonstrate the performance of RIM on some examples. All the computation is in Matlab on a 3G Hz i7 Macbook Pro with 16GB 1600 MHz DDR3 memory.

Remark 5.1. Reference eigenvalues means finite element approximations of the transmission eigenvalues obtained with the matlab "eigs" command for (4). 


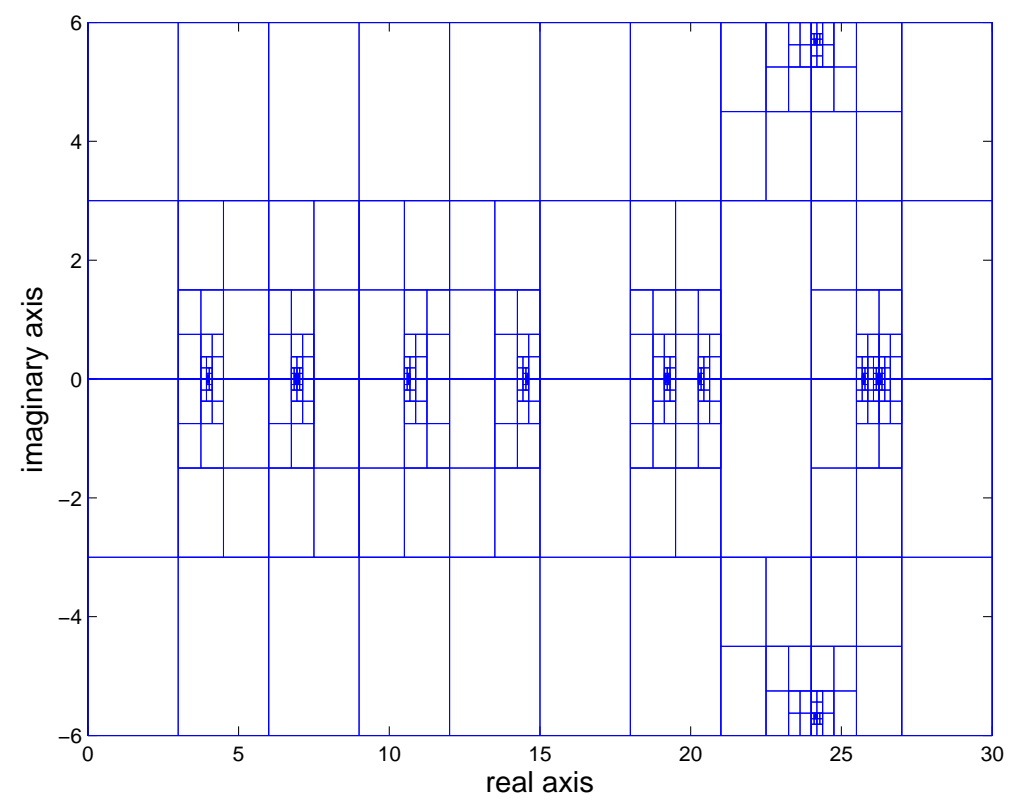

Figure 2: Exploration pattern for RIM on $S=[0,30] \times[-6,6]$ for Example 1: 16 clustered eigenvalues.

\section{$5.1 \quad$ Effectiveness}

Example 1: A triangular mesh with $h \approx 0.05$ generates two $1018 \times 1018$ matrices $A$ and $B$ for the transmission eigenvalue problem for a disc with radius $1 / 2$ and index of refraction $n(x)=16$. With $\epsilon=10^{-3}$ and $S=[3,9] \times[-3,3]$ RIM successfully returns 3 eigenvalues

$$
\begin{aligned}
& \lambda_{1}^{\text {RIM }}=\left(3.994629 \pm 10^{-3}\right) \pm 10^{-3} i \\
& \lambda_{2}^{\text {RIM }}=\left(6.935059 \pm 10^{-3}\right) \pm 10^{-3} i \\
& \lambda_{3}^{\text {RIM }}=\left(6.939941 \pm 10^{-3}\right) \pm 10^{-3} i
\end{aligned}
$$

matching the reference values $\lambda_{1}=3.994539, \lambda_{2}=6.935054, \lambda_{3}=6.939719$. With $\epsilon=10^{-3}$ and $S=[22,25] \times[-8,8] \mathbf{R I M}$ returns

$$
\begin{aligned}
& \lambda_{1}^{\text {RIM }}=\left(24.158813 \pm 10^{-3}\right)-\left(5.690308 \pm 10^{-3}\right) i \\
& \lambda_{2}^{\text {RIM }}=\left(24.158813 \pm 10^{-3}\right)+\left(5.690063 \pm 10^{-3}\right) i
\end{aligned}
$$

matching the reference eigenvalues $\lambda_{1}=24.158567+5.690114 i$ and $\lambda_{2}=24.158567-5.690114 i$. Figure 2 shows how RIM explores $S=[0,30] \times[-6,6]$ and successfully isolates all 16 eigenvalues including the complex conjugate pair. 
Example 2: For the unit square with index of refraction $n(x)=16$ the matrices $A$ and $B$ are $1298 \times 1298$. With $\epsilon=10^{-3}$ and $S=[6,9] \times[-1,1]$ RIM successfully returns 3 eigenvalues

$$
\begin{aligned}
& \lambda_{1}^{\text {RIM }}=\left(6.049316 \pm 10^{-3}\right) \pm 10^{-3} i \\
& \lambda_{2}^{\text {RIM }}=\left(6.051270 \pm 10^{-3}\right) \pm 10^{-3} i \\
& \lambda_{3}^{\text {RIM }}=\left(8.368652 \pm 10^{-3}\right) \pm 10^{-3} i
\end{aligned}
$$

matching the reference values $\lambda_{1}=6.049528, \lambda_{2}=6.051180$, and $\lambda_{3}=8.368568$. With $\epsilon=10^{-3}$ and $S=[20,21] \times[-6,6] \mathbf{R I M}$ gives

$$
\begin{aligned}
& \lambda_{1}^{\text {RIM }}=\left(20.573730 \pm 10^{-3}\right)-\left(5.127441 i \pm 10^{-3}\right) i \\
& \lambda_{2}^{\text {RIM }}=\left(20.573730 \pm 10^{-3}\right)+\left(5.126465 i \pm 10^{-3}\right) i
\end{aligned}
$$

matching the reference eigenvalues $\lambda_{1}=20.573786+5.127225 i$, and $\lambda_{2}=20.573786-5.127225 i$.

Example 3: For the L-shaped domain with $n=16$

$$
((-1,1) \times(-1,1)) \backslash([0,1] \times[-1,0])
$$

the matrices $A$ and $B$ are $978 \times 978$. With $\epsilon=10^{-3}$ and $S=[2,3] \times[-1 / 2,1 / 2] \mathbf{R I M}$ gives

$$
\begin{aligned}
& \lambda_{1}^{\text {RIM }}=\left(2.210236 \pm 10^{-3}\right) \pm 10^{-3} i \\
& \lambda_{2}^{\text {RIM }}=\left(2.506683 \pm 10^{-3}\right) \pm 10^{-3} i \\
& \lambda_{3}^{\text {RIM }}=\left(2.979706 \pm 10^{-3}\right) \pm 10^{-3} i
\end{aligned}
$$

matching the reference eigenvalues $\lambda_{1}=2.210247, \lambda_{2}=2.50668$, and $\lambda_{3}=2.979671$.

Example 4: A tetrahedral mesh with $h \approx 0.05$ for the unit ball in $\mathbb{R}^{3}$ with $n=4$ gives matrices $A$ and $B$ of size $42606 \times 42606$. With $\epsilon=10^{-3}$ and $S=[10,11] \times[-1 / 2,1 / 2]$ RIM gives

$$
\begin{aligned}
& \lambda_{1}^{\text {RIM }}=\left(10.346875 \pm 10^{-3}\right) \pm 10^{-3} i \\
& \lambda_{2}^{\text {RIM }}=\left(10.353125 \pm 10^{-3}\right) \pm 10^{-3} i \\
& \lambda_{3}^{\text {RIM }}=\left(10.371875 \pm 10^{-3}\right) \pm 10^{-3} i
\end{aligned}
$$

matching the reference values $\lambda_{1}=10.345551, \lambda_{2}=10.357927$, and $\lambda_{3}=10.369776$. Note, these are the squares of the values on page 4 of [18].

Example 5: For the unit cube in $\mathbb{R}^{3}$ with $n=16$ a tetrahedral mesh with $h \approx 0.05$ generates a $46735 \times 46735$ generalized eigenvalue problem. With $\epsilon=10^{-3}$ and $S=[4,5] \times[-1 / 2,1 / 2] \mathbf{R I M}$ gives

$$
\lambda_{1}^{\text {RIM }}=\left(4.328125 \pm 10^{-3}\right) \pm 10^{-3} i
$$

matching the reference value $\lambda_{1}=4.328288$.

Example 6: The domain

$$
((0,1) \times(0,1) \times(0,1)) \backslash([0,1 / 2] \times[0,1 / 2] \times[0,1 / 2])
$$

with $n=16$ produces a $13335 \times 13335$ generalized eigenvalue problem. With $\epsilon=10^{-3}$ and $S=[4,5] \times[-1 / 2,1 / 2] \mathbf{R I M}$ gives

$$
\begin{aligned}
& \lambda_{1}^{\text {RIM }}=\left(12.249725 \pm 10^{-3}\right) \pm 10^{-3} i \\
& \lambda_{2}^{\text {RIM }}=\left(13.102753 \pm 10^{-3}\right) \pm 10^{-3} i
\end{aligned}
$$

matching the reference eigenvalues $\lambda_{1}=12.249750$, and $\lambda_{2}=13.102771$.

RIM returns all the eigenvalues in the test regions correctly for all of these $2 \mathrm{D}$ and $3 \mathrm{D}$ examples. 


\subsection{Robustness}

Our quadrature (one point on each side) and randomization (one single normalized random vector) are notably crude compared to other contour integral based algorithms. The robustness of RIM is demonstrated by comparing the indicators obtained for various search regions with different random vectors. For Example 1 we consider two regions which contain eigenvalues.

$$
S_{1}=[3.9,4.1] \times[-0.1,0.1] \quad \text { and } \quad S_{2}=[24.1,24.2] \times[5.6,5.7]
$$

and two regions that do not contain eigenvalues

$$
S_{5}=[3.7,3.9] \times[-0.1,0.1] \quad \text { and } \quad S_{6}=[24.0,24.1] \times[5.6,5.7]
$$

For Example 2 we consider two regions that contain eigenvalues

$$
S_{3}=[6.04,6.06] \times[-0.01,0.01] \quad \text { and } \quad S_{4}=[20.5,20.6] \times[5.1,5.2]
$$

along with two regions which do not contain eigenvalues

$$
S_{7}=[6.02,6.04] \times[-0.01,0.01] \quad \text { and } \quad S_{8}=[20.4,20.5] \times[5.1,5.2] .
$$

In each case the indicators are computed with 100 different random vectors. the results are in Tables 1 and 2 .

Table 1: Indicators for regions with eigenvalues.

\begin{tabular}{rrrrr}
\hline$S$ & average & $\min$ & $\max$ & std. dev. \\
\hline$S_{1}$ & 0.63662546 & 0.63662432 & 0.63662669 & $2.42494379 \mathrm{e}-07$ \\
$S_{2}$ & 0.82076270 & 0.82076270 & 0.82076270 & $3.48933530 \mathrm{e}-11$ \\
$S_{3}$ & 0.63667811 & 0.63662296 & 0.63674302 & $4.23597573 \mathrm{e}-05$ \\
$S_{4}$ & 0.53606809 & 0.53606809 & 0.53606809 & $5.68226051 \mathrm{e}-11$ \\
\hline
\end{tabular}

Table 2: Indicators for regions with no eigenvalues.

\begin{tabular}{rrrrr}
\hline$S$ & average & $\min$ & $\max$ & std. dev. \\
\hline$S_{5}$ & 0.04778437 & 0.04778398 & 0.04778539 & $1.48221826 \mathrm{e}-07$ \\
$S_{6}$ & 0.02227906 & 0.02227906 & 0.02227906 & $6.92810353 \mathrm{e}-12$ \\
$S_{7}$ & 0.04143107 & 0.03354195 & 0.04701297 & $4.44534110 \mathrm{e}-03$ \\
$S_{8}$ & 0.01615294 & 0.01615294 & 0.01615294 & $4.94631163 \mathrm{e}-11$ \\
\hline
\end{tabular}

Remark 5.2. Tables 1 and 2 show that one random vector is adequate to distinguish the presence of eigenvalues. The indicators in Table 1 are always larger than 0.1 while those in Table 2 are all less than 0.1. Note, unlike subspace methods RIM does not resolve the dimension of the enclosed eigenspace. 
A final question is what happens when there is an eigenvalue on the edge or corner of $S$. The robustness of RIM is again demonstrated on various regions for Example 1. The test regions and indicators for a single trial are shown in Table 3. There is a real eigenvalue of 3.994690 on the shared edge between $S_{13}$ and $S_{14}$ and a complex eigenvalue of $24.158567+5.690114 i$ on the shared edge between $S_{15}$ and $S_{16}$.

Remark 5.3. Table 3 shows that in these trials even eigenvalues on the edge of $S$ are assigned to at least one region. This means that the eigenvalue is caught and tracked as the regions are further refined.

Table 3: Indicators for eigenvalues on the edge of $S$.

\begin{tabular}{lr}
\hline domain & indicator \\
\hline$S_{13}=[3.99,4.00] \times[-0.01,0.00]$ & 0.52275012 \\
$S_{14}=[3.99,4.00] \times[0.00,0.01]$ & 0.52275012 \\
$S_{15}=[24.158813,24.17] \times[5.68,5.70]$ & 0.48810370 \\
$S_{16}=[24.15,24.158813] \times[5.68,5.70]$ & 0.08569820 \\
\hline
\end{tabular}

Finally we consider an eigenvalue on the corner of $S$. Table 4 shows the results for an eigenvalue at the common corners of the four search regions $S_{17}, S_{18}, S_{19}$, and $S_{20}$ and the four search regions $S_{21}, S_{22}, S_{23}$, and $S_{24}$.

Remark 5.4. Table 4 shows that in these trials eigenvalues on a corner of $S$ are assigned to at least one region. Again, this means that the eigenvalue is caught and tracked as the regions are further refined.

Table 4: Indicators when the eigenvalue is a corner of $S$.

\begin{tabular}{lr}
\hline domain & indicator \\
\hline$S_{17}=[3.994539,4.01] \times[-0.01,0.0]$ & 0.70164096 \\
$S_{18}=[3.98,3.994539] \times[-0.01,0.0]$ & 0.91502267 \\
$S_{19}=[3.98,3.994539] \times[0.00,0.01]$ & 0.25047335 \\
$S_{20}=[3.994539,4.01] \times[0.00,0.01]$ & 0.25047335 \\
$S_{21}=[24.152,24.158567] \times[5.688,5.690114]$ & 0.43892705 \\
$S_{22}=[24.152,24.158567] \times[5.690114,5.700]$ & 0.12732395 \\
$S_{23}=[24.158567,24.161] \times[5.690114,5.700]$ & 0.12732395 \\
$S_{24}=[24.158567,24.161] \times[5.688,5.690114]$ & 0.19531957 \\
\hline
\end{tabular}

The threshold value $\delta_{0}=0.1$ was chosen based on experimentation and is important for the robustness of RIM. Eigenvalues close to $\Gamma$ introduce quadrature errors and potentially poorly conditioned linear solves. The above examples show that the choice of 0.1 is effective even in these extreme cases. 


\subsection{Self-correction Property}

The low threshold choice of 0.1 is designed to avoid missing any eigenvalues while taking advantage of a self-correcting property of RIM. When $S$ does not contain any eigenvalue but has a nearby eigenvalue quadrature error or an ill conditioned linear system might cause RIM to mistakenly believe that there is an eigenvalue and subdivide the region. Fortunately, as we will see after a few subdivisions, RIM discards these regions. We demonstrate this interesting self-correcting property using two examples.

We use matrices $A$ and $B$ from Example 1 and focus on the eigenvalue 3.994539. There is no eigenvalue in the initial search region $S=[4.0,4.2] \times[0,0.2]$ but the eigenvalue 3.994539 is just outside $S$. At first, RIM computes the indicator value

$$
0.11666587
$$

which suggests that $S$ may contain an eigenvalue. $S$ is partitioned into the four rectangles

$$
\begin{array}{ll}
S_{1}^{1}=[4.0,4.1] \times[0,0.1], & S_{2}^{1}=[4.0,4.1] \times[0.1,0.2], \\
S_{3}^{1}=[4.1,4.2] \times[0,0.2], & S_{4}^{1}=[4.1,4.2] \times[0.1,0.2]
\end{array}
$$

with indicators

$$
\begin{aligned}
& \delta_{S_{1}^{1}}=0.10687367, \quad \delta_{S_{2}^{1}}=0.00609138, \\
& \delta_{S_{3}^{1}}=0.00561028, \quad \delta_{S_{4}^{1}}=0.00182170 .
\end{aligned}
$$

RIM discards $S_{2}^{1}, S_{3}^{1}$, and $S_{4}^{1}$ and retains $S_{1}^{1}$ as admissible. The four rectangles by partitioning $S_{1}^{1}$ are

$$
\begin{aligned}
& S_{1}^{2}=[4.0,4.05] \times[0.0,0.05], \quad S_{2}^{2}=[4.0,4.05] \times[0.05,0.10], \\
& S_{3}^{2}=[4.05,4.10] \times[0.0,0.05], \quad S_{4}^{2}=[4.05,4.10] \times[0.05,0.10]
\end{aligned}
$$

with indicators

$$
\begin{aligned}
& \delta_{S_{1}^{2}}=0.08957100, \quad \delta_{S_{2}^{2}}=0.00579253, \\
& \delta_{S_{3}^{2}}=0.00494816, \quad \delta_{S_{4}^{2}}=0.00169435
\end{aligned}
$$

at which RIM will correctly discard all the regions. If the threshold were lower say 0.08 RIM would divide $S_{1}^{2}$ into

$$
\begin{gathered}
S_{1}^{3}=[4.0,4.025] \times[0,0.025], \quad S_{2}^{3}=[4.0,4.025] \times[0.025,0.05], \\
S_{3}^{3}=[4.025,4.05] \times[0,0.025], \quad S_{4}^{3}=[4.025,4.05] \times[0.025,0.05],
\end{gathered}
$$

with the following lower indicators

$$
\begin{aligned}
& \delta_{S_{1}^{3}}=0.06258907, \quad \delta_{S_{2}^{3}}=0.00519080, \\
& \delta_{S_{3}^{3}}=0.00388825, \quad \delta_{S_{4}^{3}}=0.00146650 .
\end{aligned}
$$

A search region $S=[24.16,24.96] \times[5.30,6.10]$ near the complex eigenvalue $24.158567+5.690308 i$ also generates additional evaluations before correctly concluding that there is no eigenvalue in $S$. Table. 5 shows the generated subregions and indicators. 
Table 5: Subregions and indicators for $S=[24.16,24.96] \times[5.30,6.10]$.

\begin{aligned} & \hline$S_{1}^{1}=[24.16,24.56] \times[5.30,5.70] 0.825 \\ & S_{2}^{1}=[24.16,24.56] \times[5.70,6.10] 0.195 \\ & S_{3}^{1}=[24.56,24.96] \times[5.30,5.70] 5.418 \mathrm{e}-11 \\ & S_{4}^{1}=[24.56,24.96] \times[5.70,6.10] 4.119 \mathrm{e}-11 \\ &$\hline$S_{1}^{2}=[24.16,24.36] \times[5.30,5.50] 9.216 \mathrm{e}-11 \\ & S_{2}^{2}=[24.16,24.36] \times[5.50,5.70] 0.368 \\ & S_{3}^{2}=[24.36,24.56] \times[5.30,5.50] 8.712 \mathrm{e}-14 \\ & S_{4}^{2}=[24.36,24.56] \times[5.50,5.70] 5.870 \mathrm{e}-11 \\ &$\hline$S_{1}^{3}=[24.16,24.26] \times[5.50,5.60] 1.742 \mathrm{e}-11 \\ & S_{2}^{3}=[24.16,24.26] \times[5.60,5.70] 0.781 \\ & S_{3}^{3}=[24.26,24.36] \times[5.50,5.60] 1.476 \mathrm{e}-13 \\ & S_{4}^{3}=[24.26,24.36] \times[5.60,5.70] 6.755 \mathrm{e}-11 \\ &$\hline$S_{1}^{4}=[24.16,24.21] \times[5.60,5.65] 6.558 \mathrm{e}-10 \\ & S_{2}^{4}=[24.16,24.21] \times[5.65,5.70] 0.280 \\ & S_{3}^{4}=[24.21,24.26] \times[5.60,5.65] 1.378 \mathrm{e}-13 \\ & S_{4}^{4}=[24.21,24.26] \times[5.65,5.70] 8.229 \mathrm{e}-11 \\ &$\hline$S_{1}^{5}=[24.16,24.185] \times[5.65,5.675] 1.159 \mathrm{e}-08 \\ & S_{2}^{5}=[24.16,24.185] \times[5.675,5.70] 0.156 \\ & S_{3}^{5}=[24.185,24.21] \times[5.65,5.675] 4.000 \mathrm{e}-13 \\ & S_{4}^{5}=[24.185,24.21] \times[5.675,5.70] 8.648 \mathrm{e}-11 \\ &$\hline$S_{1}^{6}=[24.16,24.185] \times[5.65,5.675] 5.574 \mathrm{e}-06 \\ & S_{2}^{6}=[24.16,24.1725] \times[5.6875,5.70] 0.095 \\ & S_{3}^{6}=[24.185,24.21] \times[5.65,5.675] 4.304 \mathrm{e}-12 \\ & S_{4}^{6}=[24.185,24.21] \times[5.675,5.70] 2.628 \mathrm{e}-11 \\ &$\hline\end{aligned}

\subsection{Close eigenvalues}

RIM separates nearby eigenvalues if $\epsilon$ is less than the distance between them. For Example 1, there are two close eigenvalues

$$
\lambda_{1}=6.935054, \quad \lambda_{2}=6.939719 .
$$

With $\epsilon=3.0 \times 10^{-2}, \mathbf{R I M}$ fails to separate the eigenvalues and outputs only one rectangle center for the cluster

$$
\lambda_{1}^{\mathbf{R I M}}=6.942500 \pm 3 \times 10^{-2}(1 \pm i) .
$$

However, with $\epsilon=10^{-4}$, RIM separates the eigenvalues correctly

$$
\begin{aligned}
& \lambda_{1}^{\text {RIM }}=6.935127 \pm 10^{-4}(1 \pm i), \\
& \lambda_{2}^{\text {RIM }}=6.939717 \pm 10^{-4}(1 \pm i) .
\end{aligned}
$$

\section{Discussions and future works}

This paper proposes a novel recursive integral method RIM for eigenvalue problems and demonstrates its ability to robustly compute transmission eigenvalues. The method isolates all the eigen- 
values in a region without a priori spectral information. The key difference between RIM and other contour integral based methods is that RIM only tests if a region contains eigenvalues or not.

We plan to extend RIM to other eigenvalue problems, study the accuracy requirements of the linear solver and the quadrature rule, and implement a parallel version of RIM.

\section{Acknowlegement}

The work of J. Sun is supported in part by NSF DMS-1521555.

\section{References}

[1] J. An and J. Shen, A Fourier-spectral-element method for transmission eigenvalue problems. J. Sci. Comput., 57 (2013), 670-688.

[2] A.P. Austin, P. Kravanja, and L.N. Trefethen, Numerical algorithms based on analytic function values at roots of unity. SIAM J. Numer. Anal. 52 (2014), no. 4, 1795-1821.

[3] W.J. Beyn, An integral method for solving nonlinear eigenvalue problems. Linear Algebra Appl. 436 (2012), no. 10, 3839-3863.

[4] F. Cakoni, D. Colton, and H. Haddar, On the determination of Dirichlet and transmission eigenvalues from far field data, C. R. Acad. Sci. Paris, Ser. I, 348 (2010), 379-383.

[5] F. Cakoni, D. Colton, P. Monk, and J. Sun, The inverse electromagnetic scattering problem for anisotropic media, Inverse Problems, 26 (2010), 074004.

[6] F. Cakoni and H. Haddar, Transmission Eigenvalues in Inverse Scattering Theory, Inside Out II, G. Uhlmann editor, MSRI Publications, Vol. 60, 527-578, 2012.

[7] F. Cakoni, P. Monk, and J. Sun, Error analysis of the finite element approximation of transmission eigenvalues. Comput. Methods Appl. Math., 14 (2014), no. 4, 419-427.

[8] D. Colton and R. Kress, Inverse Acoustic and Electromagnetic Scattering Theory, 3rd ed., Springer-Verlag, New York, 2013.

[9] D. Colton, P. Monk, and J. Sun, Analytical and Computational Methods for Transmission Eigenvalues. Inverse Problems, 26 (2010), no. 4, 045011.

[10] A. Cossonniére and H. Haddar, Surface integral formulation of the interior transmission problem. J. Integral Equations Appl., 25 (2013), no. 3, 341-376.

[11] D. Gintides and N. Pallikarakis, A computational method for the inverse transmission eigenvalue problem. Inverse Problems, 29 (2013), no. 10, 104010.

[12] G. Hsiao, F. Liu, J. Sun, and L. Xu, A coupled BEM and FEM for the interior transmission problem in acoustics. J. of Comp. and Applied Math., 235 (2011), no. 17, 5213-5221. 
[13] S. Goedecker, Linear scaling electronic structure methods, Rev. Modern Phys., 71 (1999), $1085-1123$.

[14] X. Ji and J. Sun, A multi-level method for transmission eigenvalues of anisotropic media. J. Comput. Phys., Vol. 255 (2013), 422-435.

[15] X. Ji, J. Sun, and T. Turner, A mixed finite element method for Helmholtz Transmission eigenvalues. ACM Trans. Math. Software, 8 (2012), no.4, Algorithm 922.

[16] X. Ji, J. Sun, and H. Xie, A multigrid method for Helmholtz transmission eigenvalue problems. J. Sci. Comput., Vol. 60 (2014), Iss. 3, 276-294.

[17] T. Kato, Perturbation Theory of Linear Operators, Classics in Mathematics, Springer-Verlag, Berlin, 1995.

[18] A. Kleefeld, A numerical method to compute interior transmission eigenvalues. Inverse Problems, 29 (2013), 104012.

[19] A. Lechleiter and M. Rennoch. Inside-outside duality and the determination of electromagnetic interior transmission eigenvalues. SIAM J. Math. Anal., 47 (2015), no. 1, 684-705.

[20] T. Li, W. Huang, W.W. Lin, and J. Liu, On Spectral Analysis and a Novel Algorithm for Transmission Eigenvalue Problems. J. Sci. Comput., 64 (2015), no. 1, 83-108.

[21] P. Monk and J. Sun, Finite element methods of Maxwell transmission eigenvalues. SIAM J. Sci. Comput. 34 (2012), B247-B264.

[22] J. Osborn, Spectral approximation for compact operators. Math. Comp., 29 (1975), 712-725.

[23] E. Polizzi, Density-matrix-based algorithms for solving eigenvalue problems. Phys. Rev. B., 79(2009), 115112 .

[24] T. Sakurai and H. Sugiura, A projection method for generalized eigenvalue problems using numerical integration. J. Comput. Appl. Math., 159 (2003), no. 1, 119-128.

[25] J. Sun, Estimation of transmission eigenvalues and the index of refraction from Cauchy data. Inverse Problems, 27 (2011), 015009.

[26] J. Sun, Iterative methods for transmission eigenvalues. SIAM J. Numer. Anal., 49 (2011), no. $5,1860-1874$.

[27] J. Sun, An eigenvalue method using multiple frequency data for inverse scattering problems. Inverse Problems, 28 (2012), 025012.

[28] J. Sun and L. Xu, Computation of the Maxwell's transmission eigenvalues and its application in inverse medium problems. Inverse Problems, 29 (2013), 104013.

[29] J. Sun and A. Zhou, Finite element methods for eigenvalue problems. CRC Press, Taylor \& Francis Group, Boca Raton, London, New York, 2016. 
[30] P. Tang and E. Polizzi, FEAST as a subspace iteration eigensolver accelerated by approximate spectral projection. SIAM J. Matrix Anal. Appl. 35 (2014), no. 2, 354-390.

[31] G. Yin, A contour-integral based method for counting the eigenvalues inside a region in the complex plane. arXiv:1503.05035

[32] G. Yin, R. Chan, and M. Yeung, A FEAST algorithm with oblique projection for generalized eigenvalue problems. arXiv:1404.1768

[33] Y. Yang, H. Bi, H. Li, and J. Han, Mixed methods for the Helmholtz transmission eigenvalues. SIAM J. Sci. Comput., 38(3), A1383-A1403.

[34] X. Wu and W. Chen, Error estimates of the finite element method for interior transmission problems. J. Sci. Comput., 57 (2013), 331-348.

[35] F. Zeng, J. Sun and L. Xu, A spectral projection method for transmission eigenvalue problem. Science China Mathematics, Vol. 59, Iss. 8, 1613-1622, 2016 